\title{
Carlos Gutiérrez-Noriega y su contribución a la Escuela Médica Peruana
}

\author{
SALOMÓN AYALA ${ }^{1}$, PEDRO ARELLANO ${ }^{1}$ \\ ${ }^{1}$ Sección Farmacología. Facultad de Medicina, Universidad nacional Mayor de San Marcos.
}

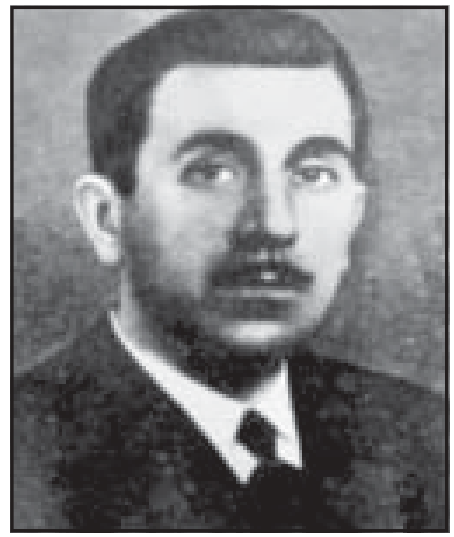

Dr. Carlos Gutierrez Noriega

1906-1950

\begin{abstract}
Resumen
El estudio presenta una aproximación a la obra científica e intelectual del maestro Carlos GutiérrezNoriega, quien fuera un destacado docente de la Facultad de Medicina de San Fernando, fundador de la farmacología en el Perú e iniciador del estudio del carácter nacional de la especialidad. Se da a conocer aspectos de su labor docente, su destacada contribución científica original en los campos de psiquiatría experimental, etnofarmacología y farmacología general, así como, sus aportes sobre cocaísmo, cocainismo, indigenismo y estudios cervantinos. Resalta los valores de su brillante trayectoria como fuerza vital en el desarrollo institucional universitario.

Palabras clave: Historia de la medicina, Perú; Gutiérrez Noriega, Carlos; farmacología.

\section{Carlos Gutierrez-Noriega and his contribution to the Peruvian Medical Shool} Abstract

This study presents an approach to the scientific and intellectual work of master Carlos GutiérrezNoriega whose labor developed during the 30's and 40's, period of major development and social genuineness of Peruvian Medicine. Founder of the Pharmacology in Peru and initiator of this specialty national character study. The review focuses his teaching labor and outstanding scientific contribution in the fields of experimental psychiatry, ethnopharmacology and general pharmacology, as well as, his investigations on cocaism and cocaine, his studies about indigenousness and the Cervantes role in medicine. It emphasizes his brilliant path values as a vital force in the university institutional development.
\end{abstract}

Key words: History of medicine, Peru; Gutiérrez Noriega, Carlos; pharmacology.

\footnotetext{
Correspondencia:

Dr. Dr. Salomón Ayala Pío

Facultad de Medicina - UNMSM

Av. Grau 755. Lima 1, Perú.

E-mail: sayalap@sanfer.unmsm.edu.pe
} 


\section{INTRODUCCIÓN}

Casi olvidado por el imaginario cultural de la Medicina Peruana y, habiendo transcurrido medio siglo de la muerte del maestro Carlos Gutiérrez-Noriega, el siguiente ensayo tiene por objetivo principal dar a conocer al colectivo médico y social una aproximación del aporte de su labor científica e intelectual.

El maestro Carlos Gutiérrez-Noriega nació en San Pedro de Lloc (Pacasmayo), el 3 de junio de 1906, hijo del señor Carlos Gutiérrez Hidalgo y de la señora Concepción Noriega. Cursó sus estudios primarios en la capital provincial de Pacasmayo, en el Centro Escolar 231, e hizo sus estudios secundarios en el Colegio Nacional de San Juan de Trujillo.

\section{ESTUDIANTE DE MEDICINA}

Ingresó a la Universidad Nacional Mayor de San Marcos el año 1924. Posteriormente, fue elegido Presidente del Centro de Estudiantes de Medicina, el año 1931, año en que la Junta de Gobierno de Sánchez Cerro promulgó el Estatuto Universitario de 1931, aplicando la «Reforma Universitaria». Durante este período realizó sus primeros trabajos científicos, resaltando "Psicoanálisis de la cultura", publicada en la Revista de los Estudiantes de Medicina, en 1932, de la cual llegó a ser director. Debido al descontento estudiantil, la agitación y el receso de la Universidad (1932 a 1935), GutiérrezNoriega cursó el sétimo año de Medicina en la Escuela de Medicina de la Universidad de Chile. El año 1935, opta el grado de Bachiller en Medicina y se titula de Médico Cirujano con la tesis: "Historial y tratamiento de una neurosis: Homosexualidad femenina y complejo caracterológico ligado al erotismo oral" ${ }^{(1)}$.

\section{DOCENTE SAN FERNANDINO}

Inició su carrera docente desde la época de estudiante de medicina, en la categoría de Ayudante de la Cátedra de Fisiología General y
Humana, labor que continuaría toda su vida; luego, pasó a ser Jefe de Trabajos Experimentales.

Graduado de Médico, en 1935, ingresa a la cátedra de Farmacología como Catedrático Auxiliar Interino, en 1937, cuyo encargado era Américo Garibaldi, quien había remplazado por dos años a Guillermo Almenara, quien, a su vez, se desempeñó como encargado del curso desde 1928 hasta 1935. Ninguno de sus antecesores en la cátedra, desde la época de Julián Arce -quien inició el curso de Farmacología en 1920-, fue farmacólogo, siendo la cátedra realizada por especialistas de otras áreas $\left({ }^{2}\right)$.

De 1938 a 1941, se desempeñó como Catedrático Principal interino de Farmacología, época en que optó el grado de Doctor en Medicina con la Tesis: "Fisiopatología y Neurobiología de la epilepsia producida por cardiazol" $\left({ }^{3}\right)$. Realizó estudios de especialización, en 1941-1942, en el Departamento de Farmacología de Chicago University, Instituto de Neurología de la Northwestern University y North Carolina University. A su regreso de los Estados Unidos, en 1942, se desempeñó como Jefe del Departamento de Química y Farmacología del Instituto Nacional de Higiene y Salud Pública, dirigida por Telémaco Battistini.

En una asamblea emocionante del Centro de Estudiantes de Medicina, en 1946, fue nombrado Delegado de la Facultad de Medicina ante el Consejo Universitario. Este año plasma su esfuerzo docente en "Farmacología y sus Aplicaciones Terapéuticas", primer libro texto publicado en el Perú, para estudiantes y médicos, donde incluye su contribución al estudio de la cocaína y de la coca, cardiazol, etc. En la primera página cita: Empezaremos el estudio de nuestra medicina por el tratamiento y no por las causas, ya que la debida aplicación de los tratamientos nos conducirá, como de la mano, a desentrañar el origen mismo de las dolencias», Paracelso (Libellus Prologorum) $\left({ }^{4}\right)$. Una segunda edición corregida y aumentada fue presentada cuatro años después $\left({ }^{5}\right)$ En 1947, funda y dirige el Instituto de Farmacología y 
Terapéutica Experimental de la Universidad Nacional Mayor de San Marcos. En 1949, invitado por la Universidad de Wisconsin, dictó conferencias en la American Society of Pharmacology and Experimental Therapeutics.

\section{APORTES EN ETNOFARMACOLOGÍA, PSIQUIATRÍA EXPERIMENTAL Y FARMACOLOGÍA GENERAL}

Inició su producción etnofarmacológica en 1936, con la Vallesia dichotoma (Cuncuna), no estudiada hasta esa época, de la cual se extrajo el alcaloide vallesina, en Alemania, siendo comercializada posteriormente por Merck con el nombre de aspirodospermina, como relajante uterino $\left({ }^{6}\right)$. Junto con Humberto Rotondo y F. Alarco, estudió la farmacodinamia de la Buttneria hirsuta. También realizó investigaciones de otras plantas medicinales, como Ficus antihelmíntica, Tabernae montana (Sanango), Ephedra americana, efectuados en animales de experimentación.

En el campo de la psiquiatría experimental, hizo numerosas investigaciones sobre neurosis experimental en animales, particularmente en lo referente a la catatonía. Asociado con Cruz, en seres humanos realizó estudios de plantas alucinógenas, como el Toe (Datura suaveolens), Ayahuasca (Banisteriopsis caapi) y la mescalina, que obtiene del San Pedro (Opuntia cilíndrica o Trichocereus pachanoi) $\left(^{7,8}\right)$. Los estudios de Meduna (1934), sobre la aplicación de los estimulantes en dosis convulsivas en las enfermedades mentales partiendo de una hipótesis del antagonismo entre epilepsia y esquizofrenia, estimularon estudios en esa línea, como "Shock hipoglicémico en la terapéutica de la esquizofrenia", "Efectos del sulfato de benzedrina en los enfermos mentales". En "Contribución al tratamiento de la esquizofrenia con insulina" $\left({ }^{9}\right)$, colaboró con Honorio Delgado, con quien mantuvo una rivalidad científica y social. En esa época, Sal y Rosas investigaba sobre el choque cardiazólico y electrochoque en pacientes esquizofrénicos, epilépticos y no epilépticos.
Sus trabajos sobre el Cardiazol los inició en 1938, con "Acerca del origen y mecanismo de las convulsiones producidas por el cardiazol en gatos descerebrados", seguido de numerosos estudios a través de los años $\left({ }^{10}\right)$, llegando a 30 artículos publicados. Trató de desentrañar los fenómenos relacionados con la epilepsia $\left({ }^{11}\right)$. En "Epilepsia producida por cardiazol en perros", trabajó con 50 perros descerebrados, divididos en bulbares, protuberanciales y mesencefálicos. Fascinado con el tema, continuó con experimentos en aves, reptiles, batracios y peces. Además, otros fármacos fueron objeto de sus investigaciones, como la nicotina, fármacos colinérgicos, vagotonina, yohimbina, morfina, adrenalina, benzedrina, coramina, estricnina, bulbocapnina, analépticos, fenobarbital.

\section{CONTRIBUCIÓN AL ESTUDIO SOBRE COCAÍSMO Y COCAINISMO}

Gutierrez Noriega inició las primeras investigaciones experimentales relacionadas con el consumo de coca en el Perú, estableciendo una nueva etapa en el conocimiento científico y cultural de este ancestral hábito. Inició sus investigaciones sobre la coca en 1937, con "Acción de la cocaína y de los extractos de la coca sobre la excitabilidad de los centros bulbares", presentado en las Jornadas de Psiquiatría Panamericana en Santiago de Chile. Luego, en colaboración con Rotondo, descubrió la catatonía experimental producida por cocaína y su reversión por los barbitúricos sobre la resistencia a la fatiga en el perro. En total publicó 26 artículos y un libro -en colaboración con Zapata- titulado "Estudios sobre la coca y cocaína en el Perú”, en 1947 (Figura 1). Respecto a los efectos del coqueo sobre la actividad mental, refiere que las alteraciones agudas que se manifiestan son excitación mental, en especial modificaciones de las percepciones, de la afectividad y del pensamiento, que son comunes a los sujetos habituados y no habituados. En las alteraciones crónicas en sujetos habituados a grandes dosis, se manifiestan en forma permanente e 
independiente de los períodos de coqueo y consisten en deterioro de la inteligencia de la memoria y de la personalidad. En estas alteraciones, podrían intervenir otros factores, como la desnutrición. Anotó que en las regiones en que predomina el coqueo hay un elevado porcentaje de analfabetismo ( $\left.{ }^{12}\right)$. En "El cocaísmo y la alimentación en el Perú" $\left({ }^{13}\right)$, indica: "La coca - droga que actúa como un narcótico de las sensaciones vitales, pues suprime el hambre, la fatiga de los organismos debilitados, la sed, el frío y las mas elementales aspiraciones humanas- fue en estas circunstancias un factor indispensable para adaptar al organismo a tan deficientes y anómalas condiciones de vida. Esta droga ha actuado como un extraordinario auxiliar del pueblo andino durante cuatro siglos, para sobrellevar la miseria más extremada".Es pertinente recordar que Carlos Enrique Paz Soldán, en 1936, refería "siempre hemos considerado al coqueo indígena una de las más poderosas causas del atraso y de la miseria de la población Peruana" $\left({ }^{14}\right)$.

Junto con Santillán, Falcón, Cobo, Acosta y Solórzano, defendía la tesis de que el uso de la coca estuvo prohibido al indio peruano, antes de la conquista y que se propagó rápidamente con las duras condiciones de vida, a consecuencia de ésta; aunque hay evidencia de su uso antes de los incas, se consideraba como un privilegio del culto religioso y de la nobleza $\left({ }^{15}\right)$. Su hipótesis de trabajo, constantemente remarcada fue: "Quien busca en las drogas una compensación a las privaciones en que vive, deja de realizar los esfuerzos indispensables para satisfacer sus aspiraciones, renuncia a la lucha por la vida, adopta una actitud pasiva e indiferente y se aleja de la realidad" $\left({ }^{16}\right)$. En 1946, aparece una corriente defendiendo al cocaísmo, por lo cual intensifica los estudios, con la participación de Cruz y Ciuffardi, desde el punto de vista químico $\left({ }^{17}\right)$. Las tesis de Salomón y Chamochumbi remarcan que la alcalinización potencia la acción de los alcaloides.

En 1948, tuvo una disputa con el profesor Carlos Monge, director del Instituto de Biología
Andina, quien sostuvo que la coca es una droga necesaria para la adaptación a la vida de las alturas y que carece de toxicidad $\left({ }^{18}\right)$. En respuesta, Carlos Gutiérrez-Noriega publica "Errores sobre la interpretación del cocaísmo en las grandes alturas" $\left({ }^{19}\right)$. Posteriormente, y de manera premonitoria, analiza el problema a nivel latinoamericano $\left({ }^{20}\right)$. En 1950 , a pedido del gobierno, viene al Perú una comisión de expertos de la ONU, quienes hacen una extensa y minuciosa revisión del cocaísmo en Perú y Bolivia. En un extenso informe, dan varias conclusiones y recomendaciones, refiriéndose a los efectos perjudiciales de la masticación y relativas al mejoramiento de la alimentación, laborales, educación, etc., así como, la posibilidad de la limitación de la producción y lograr la supresión gradual del hábito de la coca ${ }^{(21)}$.

\section{CONCEPCIÓN EN ANTROPOLOGÍA CULTURAL}

En la ciencia de la cultura humana, supo vincular a la psicología y la psiquiatría y al hombre integrado a su medio y su cultura. En su fundamental "Diseño de un estudio psicológico y antropológico de la raza Amerindia”, estudió la raza aborigen peruana, señalando, entre otros aspectos, que en la psicología del pueblo indígena peruano predomina el temperamento esquizotímico con sus diferentes subgrupos y de los caracteres introvertidos y asténicos $\left({ }^{22}\right)$ Realizó estudios sobre la biotipología en el Perú antiguo $\left({ }^{23}\right)$, sobre el pensamiento mágico del antiguo Perú ${ }^{(24)}$ y de la influencia de la geografía en la concepción del mundo $\left({ }^{25}\right)$. Estudioso del indigenismo, trató de comprender los más vitales aspectos de la realidad indoamericana.

\section{ESTUDIOS CERVANTINOS}

Varios artículos fueron dedicados a la vida y obra del genio de las letras españolas, quien vivió entre 1547 y 1616. Escribió que Cervantes 


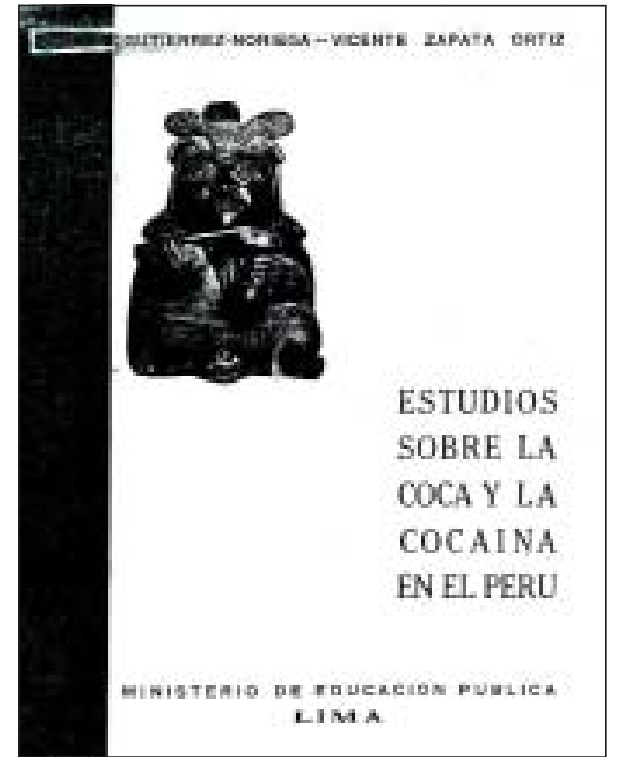

Figura 1. Libro: Estudios de la coca y cocaína en el Perú, 1947. “...se estudia de manera integral los aspectos fisiológicos, farmacológicos, patológico, psicológico y social..." (A.D.P. Introducción).

realizó observaciones psicológicas y psicopatológicas, tan originales e importantes, que permiten considerarlo como uno de los principales precursores de la psiquiatría. Fue algo indiferente a los conocimientos teóricos de la psicología antigua y su verdadera contribución viene de observaciones personales, casi de orden empírico, que él realizó por su propia cuenta $\left({ }^{26-28}\right)$. En una actuación de aniversario de la Sociedad de Neuro-Psiquiatría, en 1947, el entonces Ministro de Educación Cristóbal de Lozada y Puga, menciona: "En ella hemos podido admirar el discurso del Dr. Honorio Delgado, denso y profundo como todo lo suyo, y el notable estudio del Dr. Gutiérrez-Noriega, quien sin dejar de ser un práctico eminente y un farmacólogo de mérito y originalidad excepcional realiza la verdadera proeza -proeza de tiempo, de atención y de capacidad-de ser uno de los grandes especialistas de los estudios cervantinos" $\left({ }^{29}\right)$.

\section{PROYECCIÓN EN \\ EL IMAGINARIO CULTURAL DE LA MEDICINA PERUANA}

Procedente de un nivel socioeconómico emergente, Carlos Gutiérrez-Noriega, en su corta vida, demostró una laboriosidad infatigable y un elevado nivel de exigencia en el cumplimiento de sus metas, que logró hacerlo laborando a tiempo completo. Primer farmacólogo de formación, a diferencia de sus predecesores, entusiasmó a los jóvenes San Fernandinos y creó escuela. Su nombre se halla relacionado con carácter fundacional en todas las dimensiones de esta disciplina en el Perú.

Según Cruz, sus trabajos de investigación llegan a 120 y 10 trabajos inéditos $\left({ }^{16}\right)$, notándose que el maestro no desarrolló actividad periodística. Trabajó líneas de investigación bien definidas y no realizó trabajos aislados, sino en serie, revelando su pensamiento sistemático, fecundo y original.

Del análisis de sus publicaciones y especialmente de aquellas referidas a la enseñanza, investigación y a la posición de la farmacología en la medicina y la biología, se logra entender su amplia cultura en historia de la medicina y universal, avizorando las amplias posibilidades que ofrece la biología. Destaca a Paracelso (1493-1541) y, al parecer influenciado por Claude Bernard, Van Leewen, Leake, Gross, Dusser de Baranne y otros, en la preeminencia de los estudios de farmacología experimental. Estuvo orientado por la Escuela Universitaria Americana de Farmacología, cuya labor principal era la investigación.

En sus artículos señaló claramente la contribución y la defensa de la psiquiatría experimental como fuente de comprensión de fenómenos psicológicos, patológicos y potencialmente terapéuticos y se infiere su orientación Kraepeliana, del maestro alemán, a quien cita varias veces, como el iniciador de la fármaco-psicología. A la vez, fue el primero en estudiar el efecto psicológico de las drogas con métodos experimentales, que luego fueron 
seguidos por Klüver, Metalnikow, Weels y Huxley.

Escribió desde su época de estudiante y dirigió la Revista de los Estudiantes de Medicina. Con Telémaco Battistini fundó y editó la Revista de Medicina Experimental del Instituto Nacional de Higiene y Salud Pública, en 1944. Fue director de Actualidad Médica Peruana, donde escribió muchos de sus artículos. Con el apoyo del Rector de la Universidad, Dr. Luis Alberto Sánchez, y el Decano de la Facultad de Medicina, Dr. Sergio Bernales, fundó y dirigió el desaparecido Instituto de Farmacología y Terapéutica Experimental de la Universidad Nacional Mayor de San Marcos y la Revista de Farmacología y Medicina Experimental, cuyos nombres expresan el carácter de sus investigaciones.

Fue miembro de la Academia de Ciencias Exactas, Físicas y Naturales, de la Sociedad de Neuro-psiquiatría y Medicina Legal, de la cual fue presidente. Fue miembro del Colegio de Altos Estudios de la UNMSM, de la Sociedad Peruana de Historia de la Medicina, de la Sociedad Peruana de Filosofía, del Instituto Peruano de Cultura Hispánica, Society for Experimental Biology and Medicine, American Society for the Advancement of Science, Society Sigma $X$ y miembro honorario de la Deutsche Farmakologishes Gesellschaft de Alemania,. Estos datos fueron registrados por la bibliotecaria Sara Raez Patiño, quien recopiló los títulos, a manera de referencias bibliográficas, de casi toda la obra del maestro.

Analizando su actividad científica original y antidogmática, Rotondo dice: “...Se apartó del fácil camino trillado del que todo repite, de la erudición estéril del que nada ve con sus propios ojos y de toda discusión bizantina doctrinaria y de escuela...”.

Hacia el final de la época, junto con Weiss, Delgado, Monge, Hurtado, entre otros, hizo posible el desarrollo de la Escuela Médica Peruana (1886-1956), período en el cual San Fernando produce la mayor cantidad y calidad de conocimiento médico realizado en el país $\left({ }^{30}\right)$.
$\mathrm{Su}$ obra refleja una actividad intelectual original dirigida a interpretar distintos campos del conocimiento biológico, psicológico, experimental, indigenista, humanístico que de manera sistémica se integra en el contexto de la época. Pensador sincrético, objetivamente universal participó en la construcción de la Escuela Médica Peruana logrando un creativo desarrollo de la ciencia experimental a partir de una visión de los problemas del país.

Carlos Gutiérrez-Noriega murió en la ciudad de Pisa, Italia, cuando regresaba del Congreso Internacional de Psiquiatría en Paris, donde fue invitado a exponer sus trabajos, y luego de presentaciones en Suiza y Alemania. Su muerte fue trágica en un accidente de tránsito, viajando en compañía de Carlos Alberto Seguín, en circunstancias y contexto no bien conocidas. Sus restos fueron traídos al Perú y se le dio homenaje en el Patio de la Facultad de Medicina y en la Escuela de Ingeniería. En el homenaje, en la Sociedad de Neuropsiquiatría, que quedó escrito en las Actas de la Sociedad, se le hizo un justo reconocimiento al hombre y su obra, y el agradecimiento fue hecho por Ricardo GutiérrezNoriega, hermano del extinto.

Quedaron a cargo de la cátedra de San Fernando, Vicente Zapata Ortiz+ (después pasó a U. Cayetano Heredia) y Ramón Vargas Machuca+. También fueron sus discípulos Guillermo Cruz Sánchez+, Víctor Cárcamo Márquez+, Delgado Matallana, Pedro Arellano Jiménez y William Cárdenas Ruiz+. Los dos últimos permanecieron a Farmacología y se dedicaron a la docencia y a la investigación en plantas medicinales nativas.

Su extensa biblioteca quedó en el local de Farmacología. Hace 5 años fue trasladada al Museo de la Biblioteca de San Fernando.

\section{Agradecimiento}

Al Dr. Guillermo Contreras por sus críticas e importantes ideas. A la Biblioteca de la Facultad de Medicina UNMSM y a su bibliotecario el Sr. 
Carlos Larrea Yarlequé por su valiosa colaboración.

\section{REFERENCIAS BIBLIOGRÁFICAS}

1. Gutiérrez C. Historial y tratamiento de una neurosis: homosexualidad femenina y complejo caracterológico ligado al erotismo oral. Lima: Talleres Gráficos del Hospital "Víctor Larco Herrera"; 1936.

2. Castro R. Farmacología. En: Salaverry O (Editor). Historia de la Medicina Peruana en el siglo XX. Lima, Perú: Fondo Editorial UNMSM; 2000.

3. Gutiérrez-Noriega C. Fisiopatología y neurobiología de la epilepsia producida por cardiazol. [Tesis Doctoral]. Lima: Facultad de Medicina, Universidad Nacional Mayor de San Marcos; 1940.

4. Gutiérrez-Noriega C. Farmacología y sus aplicaciones terapéuticas Lima: Ed. Médica Peruana; 1946.

5. Gutiérrez-Noriega C. Farmacología y sus aplicaciones Terapéuticas. 2da. ed. Lima, Perú: Ed. Lumen S.A.; 1950.

6. Gutiérrez-Noriega C. Farmacología de la Vallesia dichotoma, I Principales propiedades farmacodinámica de sus extractos II Farmacodinamia de sus alcaloides. Actualidad Médica Peruana 1936;1(11):646-51.

7. Gutiérrez-Noriega C. Observaciones preliminares de la farmacodinamia del toé. Actualidad Médica Peruana 1937;(12):482-95.

8. Gutiérrez-Noriega C, Cruz G. Psicosis experimental producida por la Opuntia cylindrica. Revista de NeuroPsiquiatría 1948;11(2):155-70.

9. Delgado H., Valega J, Gutiérrez-Noriega C. Contribución al tratamiento de la esquizofrenia con insulina. Revista de Neuro-Psiquiatría 1938;1(4):463-98.

10. Gutiérrez-Noriega C. Levels of action of pentamethylenetetrazol with the Horsley-Clarke stereotxic apparatus. J Neuropath Exper Neurol 1943;2:132.

11. Gutiérrez-Noriega C. Biologie et physiologie des convulsions epileptiques. Scientia 1947;3:38-43.

12. Gutiérrez-Noriega C. Zapata V. Estudios sobre la coca y la cocaína en el Perú. Lima: Ed. Educación Artística y Extensión Cultural. Ministerio de Salud Pública; 1947.

13. Gutiérrez-Noriega C. El cocaísmo y la alimentación en el Perú. An Fac Med 1948;31(1):3-92.

14. Paz Soldán C. Situación actual de la coca Peruana. La Reforma Médica 1936;1:36.
15. Gutiérrez-Noriega C. Datos históricos sobre la habituación de la coca en el Perú. Revista de Medicina Experimental 1944;3(4):321-43.

16. Cruz, G. La obra de Carlos Gutiérrez Noriega. Revista de Neuro-Psiquiatría 1951;14(1):134-48.

17. Ciuffardi E. Dosis de alcaloides que ingieren los habituados a la coca. Revista de Farmacología y Medicina Experimental 1948;1:81-99.

18. Cabieses F. La acción antifatigante de la cocaína y la habituación de la coca en el Perú. An Fac Med 1946;29(4):316-67. (Publicado en 1948).

19. Gutiérrez-Noriega C. Errores sobre la interpretación del cocaísmo en las grandes alturas. Revista de Farmacología y Medicina Experimental 1948;1(1):100-23.

20. Gutiérrez Noriega C. Uber den Cocaismus in Sudamerika Die Pharmazie 1950;(5):317.

21. Consejo Económico Social. Informe de la Comisión de Estudio de las hojas de la Coca. New York: Informe de la ONU; 1950. p. 13-175.

22. Gutiérrez-Noriega C. Diseño de un estudio psicológico y antropológico de la raza Amerindia. Actualidad Médica Peruana 1937;3(4):159-95.

23. Gutiérrez-Noriega C. Biotipología del Perú antiguo. Actualidad Médica Peruana 1936;2(3):118-30.

24. Gutiérrez-Noriega C. El pensamiento mágico en las pinturas del antiguo Perú. Revista de Neuro-Psiquiatría 1939;2(3):427-64.

25. Gutiérrez-Noriega C. La concepción del mundo y la reacción espiritual congruentes al medio geográfico en el antiguo Perú. New York: Nueva Democracia; 1937.

26. Gutiérrez-Noriega C. Contribución de Cervantes a la Psicología y a la Psiquiatría. Revista de Neuro-Psiquiatría 1944;7(2): 149 .

27. Gutiérrez-Noriega Contribución de Cervantes a la Psicología Médica. Revista de Neuro-Psiquiatría 1946;9(2):107-19.

28. Gutiérrez-Noriega C. Psicologías anormales en la novela picaresca española del siglo de oro. Lima: Boletín del Instituto Peruano de Cultura Hispánica; 1948.

29. Gutiérrez-Noriega C. La personalidad y el carácter en la obra de Cervantes. Revista de Neuro-Psiquiatría 1947;51642.

30. Murillo J, Salaverry O, Mendoza W, Franco G, Calderón $\mathrm{N}$, Rodríguez-Tafur J. Daniel Alcides Carrión y su contribución al imaginario cultural de la Medicina Peruana. An Fac Med 2000;63(2):141-59. 